\title{
Post-Acute Sequelae of SARS-Cov-2 Infection Associating Peripheral, Autonomic and Central Nervous System Disturbances: Case Report and Review of the Literature
}

\author{
David S. Younger \\ Department of Neuroscience, City University of New York Medical School, New York NY, USA \\ Email: youngd01@nyu.edu
}

How to cite this paper: Younger, D.S. (2021) Post-Acute Sequelae of SARS-Cov-2 Infection Associating Peripheral, Autonomic and Central Nervous System Disturbances: Case Report and Review of the Literature. World Journal of Neuroscience, $11,17-21$.

https://doi.org/10.4236/wjns.2021.111003

Received: January 20, 2021

Accepted: January 30, 2021

Published: February 2, 2021

Copyright $\odot 2021$ by author(s) and Scientific Research Publishing Inc. This work is licensed under the Creative Commons Attribution International License (CC BY 4.0).

http://creativecommons.org/licenses/by/4.0/

(c) (i) Open Access

\begin{abstract}
Aim: To describe post-infectious neurological aspects of COVID-19 in a patient with progressive post-infectious peripheral (PNS), autonomic (ANS) and central nervous system (CNS) involvement due to SARS-CoV-2 infection. Background and Purpose: A variety of neurological manifestations have been described in association with Covid-19, however, progressive multisystem neurological aspects have not been described. Methods: A case report detailing the history, examination, and infectious serology associated with SARS-CoV-2, and subsequent neurodiagnostic laboratory testing and treatment. Results: Neurodiagnostic laboratory studies showed large-fiber demyelinating sensorimotor and painful small fiber sensory polyneuropathy, orthostatic hypotension, and hypometabolism of bilateral anterior and mesial temporal lobes with a possible frontal seizure focus. Treatment was initiated with high-dose immune globulin therapy. Conclusions: The combination of PNS, ANS and CNS involvement in this patient was associated with post-infectious acquired immunity to SARS-CoV-2.
\end{abstract}

\section{Keywords}

COVID-19, SARS-CoV-2, Long Hauler, Long Covid, Peripheral Neuropathy, Dysautonomia, Encephalopathy, Encephalitis, EMG NCS, Tilt Table Test, Epidermal Nerve Fibers, PET/MRI

\section{Introduction}

The 2019 novel coronavirus (2019-nCoV [COVID-19]) epidemic is caused by the novel Severe Acute Respiratory Syndrome-Coronavirus-2 (SARS-nCoV-2 or 
SARS-CoV-2). There is a recent review of acute neurological illness associated with COVID-19 [1]. Less attention has been devoted to chronic progressive neurological deficits.

\section{Materials and Methods}

The diagnosis of acute COVID-19 infection and immunity was confirmed by a positive reverse-transcriptase polymerase-chain-reaction (RT-PCR) from a nasopharyngeal swab, and acquired immunity was ascertained by the presence of elevated SARS-CoV-2 IgG-specific serum antibody. Electrodiagnostic studies (EDX) were performed in three limbs according to standard methods [2]. Epidermal nerve fiber (ENF) densities were ascertained in a 3-mm punch biopsy of skin taken from the lateral thigh and calf and placed in paraformaldehyde-lysine-periodic acid fixative and compared to age-matched controls using normative data [3] [4]. Autonomic evaluation of beat-to-beat blood pressure acquisition for testing of cardiovagal (parasympathetic) function, adrenergic (sympathetic) function of heart rate (HR) and systolic blood pressure (SBP) during rapid respiration, Valsalva maneuver and 5-minute 70-degree head up tilt (HUT) table test were performed according to standard guidelines. [5] ${ }^{18}$ Fluorodeoxyglucose (FDG) positron emission tomography fused to non-contrast magnetic resonance imaging (PET/MRI) of the brain with 3D rendering was performed using a standard protocol for autoimmune encephalopathy. Mayo Clinic (Mayo Labs, Rochester, MN) serum autoantibody (ENS2) panel screened for autoimmune encephalopathy.

\section{Results}

A 53-year-old woman tested positive for the SARS-CoV-2 in the spring 2020 associated with an upper respiratory infection and loss of taste. Later in the summer she developed an erratic pulse and BP, fatigue, neuropathic pain, weakness, and brain fog. Examination in December 2020 showed stocking sensory loss to vibration, cold temperature, and pin prick, mild weakness in strength graded $4+/ 5$ in the arms and legs with hyporeflexia. EDX showed mixed chronic distal demyelinating and axonal changes. ENF densities were in the low normal range for age in the thigh (patient value, 12.9 ENFs per mm skin; normal 21.4 $\mathrm{ENFs} / \mathrm{mm}$ ) and below the normal range for age in the calf (patient value, 8.4 ENFs/mm; normal $9.8 \mathrm{ENFs} / \mathrm{mm}$ ) without significant histologic abnormalities (Figure 1). HUT table testing showed symptomatic orthostatic intolerance associated with a sustained fall in SBP to $80 \mathrm{mmHg}$ and a compensatory HR acceleration to $121 \mathrm{bpm}$. FDG PET/MRI with 3D post-processing showed hypometabolism of bilateral anterior and mesial temporal lobes and a focal area of FDG hypometabolism in the high left paramedian superior frontal gyrus with normal cortical volumes (Figure 2(a) and Figure 2(b)). Mayo Clinic ENS2 panel showed no autoantibodies. She was started on high-dose 2 grams per kilogram of monthly intravenous immune globulin (IVIg) therapy. 


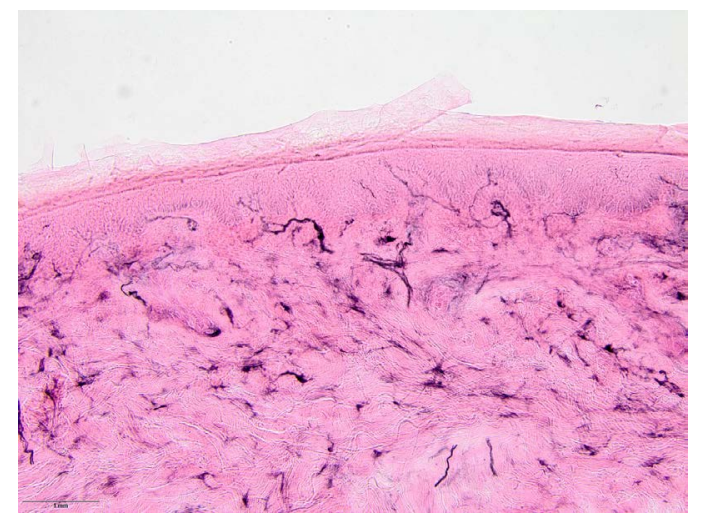

Figure 1. Left thigh epidermal nerve fiber biopsy. Immunohistochemical staining of epidermal nerve fibers (ENF) using the PGP9.5 antibody, counter-stained by Eosin. The density of ENF was $12.9 / \mathrm{mm}$. Original magnification $\times 200$.

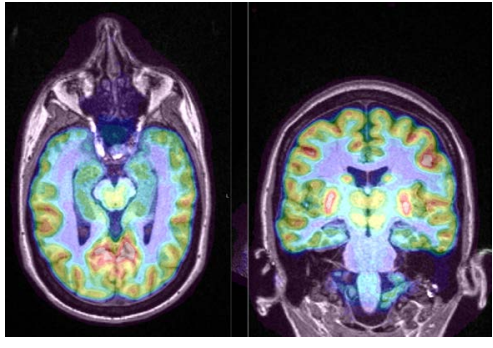

(a)

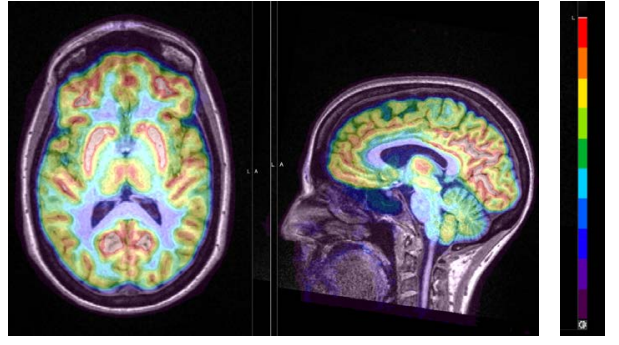

(b)

Figure 2. (a) and (b) FDG PET fused to MRI demonstrates symmetric moderate FDG hypometabolism in the medial temporal lobe structures, thalami and cerebellum (blended volumetric T1-weighted images with superimposed FDG, scale bar "red" set to right sensorimotor cortex). There was no structural MRI correlate.

\section{Discussion}

Progressive PNS, ANS and CNS involvement developed in a patient convalescing from Covid-19 illness. Although autoimmune encephalopathy was suggested by hypometabolism of bilateral mesial temporal lobes, there was no circulating autoantibody or history of seizures or seizure focus, to make a definite diagnosis as in six other reported patients who underwent PET brain imaging unfused to MRI [6]. There are several reasons to consider the contribution of immune mechanisms in Covid-19 neurological illness. Clinicopathologic studies of decedents [7] show elevated levels of circulating interleukin (IL)-6, IL-8, and tumor necrosis factor (TNF)- $\alpha$, suggesting activation of innate and adaptive immunity during the acute illness indicative of a cytokine storm associated with focal and diffuse cortical, brainstem, and leptomeningeal T-cell mediated inflammation in postmortem examination.

The parainfectious autoimmune disorder, acute inflammatory demyelinating polyradiculoneuropathy accompanies acute systemic COVID-19 illness [8]. Painful small fiber sensory polyneuropathy demonstrated on ENF studies and dysautonomia by standard testing with symptoms of brain fog were described as a post-infectious COVID-19 syndrome treated with IVIg in a similar case, 
however, EDX and PET/MRI were not reported [9]. One region of the brain that may be important in COVID-19 related neurological illness is the mesial temporal lobes and hippocampus, which were found to be hypometabolic in the present case. These structures are notable for their role in modulating mood and memory by increasing or decreasing the integration of neurons in its delicate network [10]. The finding of altered metabolism in these structures without morphological changes, which may be a potential marker of COVID-related brain dysfunction and its reversibility with immune modulatory therapy such as IVIg, would be notable achievements in understanding the mechanism of post-Covid encephalopathy.

The precise pathogenesis of the neurological illness in the present patient is not well understood, however, it is likely to be related to preceding SARS-CoV-2 infection and its direct and indirect effects on neurons. These mechanisms were captured in a recent study by Ramani and coworkers [11] employing a brain organoid model. They found increased terminal deoxynucleotidyl transferase dUTP nick end labeling (TUNEL) staining indicating deoxyribonuclease (DNA) breaks and fragmentation typical of the terminal phase of apoptosis. In addition, there was a reversible deposition of $\mathrm{pT} 231$ Tau in SARS-CoV-2 positive neurons, capable of triggering the downstream cascade of immune-inflammation, neuronal stress, and direct neurotoxicity.

The mechanism of post-infectious immunity, which appears likely in the present patients may be described by the acronym, I-Cubed $\left(\mathrm{I}^{3}\right)$ that posits the multiplier effect of infection, immunity, and inflammation, which when conditioned by environmental and genetic predisposing factors, can be the source of nervous system autoimmunity [12]. It is unlikely that the designations, Covid Long Hauler and Long Covid [13] are inclusive enough to describe this disorder, however, given the magnitude of those affected, and their congregation and self-selection among mounting Facebook Groups (https://www.facebook.com/groups/359321171725541), they may be useful cohorts for future studies (https://www.davidsyounger.com).

\section{Conflicts of Interest}

The author has not conflicts of interest to report.

\section{References}

[1] Younger, D.S. (2021) Coronavirus 2019: Clinical and Neuropathological Aspects. Current Opinion in Rheumatology, 33, 49-57. https://doi.org/10.1097/BOR.0000000000000769

[2] Ferrante, M. (2015) Chapter 5. Electrodiagnostic Studies in Motor Disorders. In: Younger, D.S., Ed., Motor Disorders, $3^{\text {rd }}$ Edition, Rothstein Publishers, Brookfield, CT, 83-107.

[3] McArthur, J.C., Stocks, E.A., Hauer, P., et al. (1998) Epidermal Nerve Fiber Density: Normative Reference Range and Diagnostic Efficiency. Archives of Neurology, 55, 1513-1520. https://doi.org/10.1001/archneur.55.12.1513 
[4] Lauria, G., Bakkers, M., Schmitz, C., et al. (2010) Intraepidermal Nerve Fiber Density at the Distal Leg: A Worldwide Normative Reference Study. Journal of the Peripheral Nervous System, 15, 202-207. https://doi.org/10.1111/j.1529-8027.2010.00271.x

[5] Cheshire, W.P., Freeman, R., Gibbons, C.H., et al. (2020) Electrodiagnostic Assessment of the Autonomic Nervous System: A Consensus Statement Endorsed by the American Autonomic Society, American Academy of Neurology, and the International Federation of Clinical Neurophysiology. Clinical Neurophysiology. https://doi.org/10.1016/j.clinph.2020.11.024

[6] Delorme, C., Paccoud, O., Kas, A., et al. (2020) COVID-19-Related Encephalopathy: A Case Series with Brain FDG-Positron-Emission Tomography/Computed Tomography Findings. European Journal of Neurology, 27, 2651-2657. https://doi.org/10.1111/ene.14478

[7] Younger, D.S. (2020) Postmortem Neuropathology in COVID-19. Brain Pathology., 23, e12915 [Epub ahead of print]. https://doi.org/10.1111/bpa.12915

[8] Caress, J.B., Castoro, R.J., Simmons, Z., et al. (2020) COVID-19-Associated Guillain-Barré Syndrome: The Early Pandemic Experience. Muscle \& Nerve, 62, 485-491. https://doi.org/10.1002/mus.27024

[9] Novak, P. (2020) Post COVID-19 Syndrome Associated with Orthostatic Cerebral Hypoperfusion Syndrome, Small Fiber Neuropathy and Benefit of Immunotherapy: A Case Report. eNeurologicalSci, 21, 100276. https://doi.org/10.1016/j.ensci.2020.100276

[10] Baptista, P. and Andrade, J.P. (2018) Adult Hippocampal Neurogenesis: Regulation and Possible Functional and Clinical Correlates. Frontiers in Neuroanatomy, 12, 44. https://doi.org/10.3389/fnana.2018.00044

[11] Ramani, A., M€uller, L., Ostermann, P.N., et al. (2020) SARS-CoV-2 Targets Neurons of 3D Human Brain Organoids. The EMBO Journal, 39, e106230. https://doi.org/10.15252/embj.2020106230

[12] Younger, D.S. (2020) Book Review: I-Cubed and the Autoimmune Brain: A Five-Step Plan. World Journal of Neuroscience, 10, 29-36. https://doi.org/10.4236/wjns.2020.101005

[13] Callard, F. and Perego, E. (2021) How and Why Patients Made Long Covid. Social Science \& Medicine, 268, 113426. https://doi.org/10.1016/j.socscimed.2020.113426 